\title{
Revising Z: Part I - logic and semantics
}

\author{
Martin C. Henson ${ }^{1}$ and Steve Reeves ${ }^{2}$ \\ ${ }^{1}$ Department of Computer Science, University of Essex, UK; \\ ${ }^{2}$ Department of Computer Science, University of Waikato, New Zealand
}

Keywords: Specification language Z; Logics of specification languages; semantics of specification languages

\begin{abstract}
This is the first of two related papers. We introduce a simple specification logic $Z_{C}$ comprising a logic and a semantics (in ZF set theory) within which the logic is sound. We then provide an interpretation for (a rational reconstruction of) the specification language $\mathrm{Z}$ within $\mathrm{Z}_{C}$. As a result we obtain a sound logic for $\mathrm{Z}$, including a basic schema calculus.
\end{abstract}

\section{Introduction}

\subsection{Background}

The specification language $\mathrm{Z}$ has been in existence, and has been very widely used, for more than a decade. There is, however, no definitive logical account of Z, although progress has been made (e.g. [WB92], [Bri95] ${ }^{1}$, [Nic95], [HM97], [Toy97], [BM96], [WD96], [Mar98]). These attempts to provide $Z$ with a logic are not accompanied by meta-mathematical results such as a soundness proof; indeed, the logic in [Nic95] (and [BM96]) is inconsistent (see [Hen98] and note that [Mar98] repairs the error).

Our aim in this paper is to establish a logic for a version of $Z$, the development and nature of which has been guided by our associated mathematical investigations. This means that we have felt it necessary to allow the mathematical investigation to form, in part, a critique of the language $\mathrm{Z}$ as it is generally

Correspondence and offprint requests to: Martin Henson, Department of Computer Science, University of Essex, Wivenhoe Park, Colchester, Essex, C04 3SQ, UK

1 This has now been superseded by a new thesis which is currently in the process of being examined. It contains a logic and is accompanied by a mathematical analysis. Brien's work is complementary to ours and will provide an opportunity for a fruitful comparative investigations in the future. 
understood. The critique, and the revisionism which accompanies it, emerges most explicitly in the companion paper [HeR99]. Our own view is that developing a logic for $\mathrm{Z}$ long after establishing its language will inevitably suggest certain areas in which the language is best adjusted. Doubtless others may disagree, at least with the particular revisions we propose but, for our part, we have our mathematical analysis to resort to in substantiating claims of conceptual simplicity and elegance, and for defending our neologisms.

We do not offer this work as a final, nor even a definitive, account but we do feel that what follows establishes a methodologically sound trajectory for further work and fruitful comparison. Our work should, therefore, be considered as complementary to, rather than as a competitor of, the work which continues on the $\mathrm{Z}$ standard (which is, in part, working towards completing a logic for standard Z) and it will be very interesting to compare that with the approach we have taken here.

In discussing a logic for $\mathrm{Z}$, it is also necessary to mention the related topic of semantics. $Z$ semantics has, since [Spi88], been in a more well-developed state than the logic. This work has been substantially revised and updated by those responsible for the relevant sections of [Nic95]. It is quite possible that the semantic perspective has been given conceptual priority by those most concerned with these matters during the last decade. Although we would wish to argue strongly against such a priority, this will not be our major concern here, and it is only our surmise that that has been, in fact, the case. However, we would wish the reader to bear the following in mind in reading the paper: by giving the logic conceptual priority we are able to enormously simplify the semantics for Z. The most important consequence of this simplicity is that we will be able to provide a soundness proof for our logic (corollaries 5.3 and 5.5 below) with a very simple and straightforward proof. This observation should be contrasted with the situation in, for example, [Nic95] where there is no soundness proof, and the prospect that it would be enormously complicated to construct.

We have not, so far, mentioned proof-tools, but as [Mar97] has accurately diagnosed, the existence of a logic for $Z$ should be considered a precondition for the proper development of proof-tools for $\mathrm{Z}$, and is, therefore, a separate, though related, research area. It is, of course, a very important area and [Mar97] provides a comprehensive review of existing work to date, the most notable of which is perhaps the shallow semantic embedding of [KSW96].

\subsection{Organisation of the paper}

In Section 2 we introduce a specification system $Z_{C}$ which is essentially a typed set theory incorporating the notion of a schema type. This system is, when compared with $\mathrm{Z}$ in its notational scope, very simple indeed. $Z_{C}$ is much more than a notation: it is a specification logic and the language is associated with both rules for determining the types of terms, rules for determining that propositions are well-formed, and rules of inference. The meta-mathematical measure we investigate is the property of syntactic consistency: we show that all provably true (proto-)propositions are well-formed. $Z_{C}$ forms an effective bridge between $\mathrm{Z}$ proper and the intended model in classical extensional set theory. In Section 3 we show how $Z_{C}$ may be interpreted quite simply in $Z F$ using, in particular, a suitable dependent product operation over a family of sets over a very small 
(in ordinal terms) universe of sets. The interpretation is shown to be suitable by means of soundness results of a standard and, in this case, very simple kind.

In Section 4 we introduce a notation which is very much closer to the $\mathrm{Z}$ familiar in the literature. We include more substantial mechanisms for the construction of propositions, sets and, in particular, a basic algebra of schema operations. This notation is also equipped with a system of rules for type assignment for terms and propositionhood, together with a logic. As before, we can show that this system is syntactically consistent with respect to type assignment and propositionhood. In Section 5 we are able to describe an interpretation for this $Z$ within the much simpler system $Z_{C}$. The interpretation has several significant features. We are able to test our interpretation by showing that it preserves the type assignment and propositionhood systems of both $\mathrm{Z}$ and $\mathrm{Z}_{C}$. Finally we are able to demonstrate that the interpretation of $\mathrm{Z}$ in $\mathrm{Z}_{C}$ is sound: it preserves entailment. By composing results we complete the paper with a soundness theorem for $Z$ in the intended model ZF.

The system we develop here is still a somewhat impoverished version of $\mathrm{Z}$, and the companion paper [HeR99] contains extensions to the full system as we have developed it.

\section{The specification logic $Z_{C}$}

In this section we shall describe a simple specification logic which we call $\mathrm{Z}_{C}$. It is based upon the notion of schema type which has been introduced in $Z$. Our strategy will be to interpret higher-level features of $\mathrm{Z}$ within this logic. The idea of interpreting the language of $\mathrm{Z}$ within a small core language is not new. The approach is novel in presenting a core specification logic and undertaking a systematic mathematical analysis.

\subsection{The language of $Z_{C}$}

We begin with types.

$$
T::=\mathbb{N}|\mathbb{P} T| T \times T \mid[D]
$$

Schema types $[D]$ are explicitly part of the type system. We take $\mathbb{N}$ rather than $\mathbb{Z}$ to be the primitive type of $Z_{C}$. This is a more natural choice from a logical point of view and we may impose conventional rules for $\mathbb{N}$ from which other derived number systems, such as $\mathbb{Z}$, may be obtained.

Declarations are very simple.

$$
D::=l: T \mid l: T ; D
$$

Declarations of the form $l: T$ are called prime declarations. The labels $l$ are constants. Prime declarations $l_{0}: T_{0}$ and $l_{1}: T_{1}$ are compatible unless $l_{0}=l_{1}$ and $T_{0} \neq T_{1}$. This idea extends naturally to schema types. We shall write $\left[D_{0}\right] \preceq\left[D_{1}\right]$ when the set of prime declarations of $D_{0}$ is a subset of that of $D_{1}$. Other meta-operations we shall need over (compatible) schema types: $\left[D_{0}\right] \backslash\left[D_{1}\right]$ is the schema type comprising all prime declarations of $\left[D_{0}\right]$ which do not occur in $\left[D_{1}\right]$. The schema type $\left[D_{0}\right] \vee\left[D_{1}\right]$ is the schema type comprising the union of the prime declarations in $D_{0}$ and $D_{1}$. As a matter of convention, which simplifies the 
presentation, we take the ground instances of meta-syntactic expressions to be all and only those for which the relevant meta-operations are well-defined. This avoids laborious notation and extra sideconditions. For example writing $T_{0} \vee T_{1}$ forces $T_{0}$ and $T_{1}$, in the context in which the expression appears, to be compatible schema types.

We also need the usual notion of substituting for a variable with a term. We denote the operation of substitution by $t_{1}\left[x / t_{0}\right]$ (in the meta-language) which means that all free occurrences of $x$ are replaced by $t_{0}$ in $t_{1}$. This is extended in the usual way to allow simultaneous substitution of several variables.

Finally, we shall introduce meta-notational conventions which require substitution for labels. For this we need the alphabet operator, defined as follows: Let $[D]=\left[\cdots l_{i}: T_{i} \cdots\right]$. Then $\alpha[D]={ }_{d f}\left\{\cdots l_{i} \cdots\right\}$ and we shall write $[\alpha[D] / t . \alpha[D]]$ to represent the family of substitutions: $\cdots\left[l_{i} / t . l_{i}\right] \cdots$

The formulae of $\mathrm{Z}_{C}$ delineate a typed predicate logic. We begin with the category which forms the basis for the language of well-typed formulae we require.

The proto-syntax of formulae is given by:

$$
P::=\perp|t=t| t \in t|\neg P| P \vee P \mid \exists z: T \bullet P
$$

The logic of $Z_{C}$ is classical and so the remaining logical connectives and the universal quantifier can be defined in terms of the above in the usual manner.

The proto-syntax of terms is as follows:

$$
\begin{aligned}
t::=x|n| & |z: T| P\}|t . l|\langle\cdots l \Rightarrow t \cdots\rangle|t .1| t .2 \\
& |(t, t)| t \uparrow[D]
\end{aligned}
$$

The last term formation operator is new and has no history in Z. We pronounce

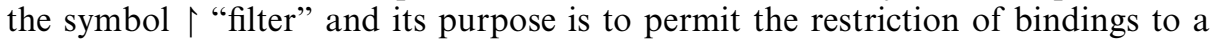
given schema type. We shall see, in Section 5, how important these filtered terms are for constructing compositional interpretations for schema expressions. Our choice of notation indicates that there is an intimate relationship between filtered terms and restricted schemas; this is made clear in section 2.5 of [HeR99].

The subcategory of numerals is as expected:

$$
n::=0 \mid \operatorname{succ} n
$$

For notational clarity we will use, in the sequel, the meta-variable $C$ to range over set-terms. That is, terms which, under the well-formation system we now turn to, have the type $\mathbb{P} T$ for some $T$.

\subsection{Type assignment and propositionhood in $Z_{C}$}

Definition 2.1. Sequents of the system have the form: $\Gamma \triangleright_{C} J$ where $J$ is a judgement that a term has a type or that a proto-formula is a proposition. We will omit the subscript on the entailment symbol, whenever the context allows, in this system and in all the other systems which follow. $\Gamma$ is a type assignment context for variables. Such contexts are understood to be partial functions from variables to types. They are extended as follows:

$$
\Gamma, x: T=_{d f} \lambda y \cdot \begin{cases}T & x=y \\ \Gamma(y) & \text { otherwise }\end{cases}
$$


For clarity of presentation we shall omit the entailment symbol and all components of contexts which are irrelevant to, or which remain unchanged by, any rule.

$$
\begin{aligned}
& \overline{\perp \text { prop }}\left(C_{\perp}\right) \quad \frac{t_{0}: T \quad t_{1}: T}{t_{0}=t_{1} \text { prop }}\left(C_{=}\right) \quad \frac{t: T \quad C: \mathbb{P} T}{t \in C \text { prop }}\left(C_{\in}\right) \\
& \frac{P \text { prop }}{\neg P \text { prop }}\left(C_{\neg}\right) \quad \frac{P_{0} \text { prop } P_{1} \text { prop }}{P_{0} \vee P_{1} \text { prop }}\left(C_{\vee}\right) \quad \frac{x: T \triangleright P \text { prop }}{\exists x: T \bullet P \text { prop }}\left(C_{\exists}\right) \\
& \overline{x: T \triangleright x: T}\left(C_{x}\right) \quad \overline{0: \mathbb{N}}\left(C_{0}\right) \quad \frac{n: \mathbb{N}}{\operatorname{succ} n: \mathbb{N}}\left(C_{s}\right) \\
& \frac{x: T \triangleright P \text { prop }}{\{x: T \mid P\}: \mathbb{P} T}\left(C_{\{\}}\right) \quad \frac{t:\left[\cdots l_{i}: T_{i} \cdots\right]}{t . l_{i}: T_{i}}(C .) \\
& \frac{\cdots t_{i}: T_{i} \cdots}{\left\langle\cdots l_{i} \Rightarrow t_{i} \cdots\right\rangle:\left[\cdots l_{i}: T_{i} \cdots\right]}\left(C_{\Rightarrow}\right) \\
& \frac{t: T_{1} \times T_{2}}{t .1: T_{1}}\left(C_{\mathrm{I}}\right) \quad \frac{t: T_{1} \times T_{2}}{t .2: T_{2}}\left(C_{2}\right) \\
& \frac{t_{0}: T_{0} \quad t_{1}: T_{1}}{\left(t_{0}, t_{1}\right): T_{0} \times T_{1}}\left(C_{0}\right) \quad \frac{t: T_{1} \quad T_{0} \preceq T_{1}}{t \uparrow T_{0}: T_{0}}\left(C_{\uparrow}\right)
\end{aligned}
$$

Lemma 2.2. (The generation lemma for $Z_{C}$ ).

(i) If $\Gamma \triangleright P_{0} \vee P_{1}$ prop then $\Gamma \triangleright P_{0}$ prop and $\Gamma \triangleright P_{1}$ prop

(ii) If $\Gamma \triangleright\{x: T \mid P\}: T_{0}$ then $\Gamma, x: T \triangleright P$ prop and $T_{0}=\mathbb{P} T$

Proof. (i) There is only one rule with a conclusion of the form $\Gamma \triangleright P_{0} \vee P_{1}$ prop, namely: $\left(C_{\vee}\right)$. (ii) Similarly.

These are two cases of a general result known as the generation lemma. There are many similar cases for all other conclusion forms and these are all proved in exactly the same manner.

\subsection{Some consequences of typechecking}

Before we move on to discuss the logic of $Z_{C}$ there are number of auxiliary results which concern the rules for proposition formation and type assignment introduced in definition 2.1.

\section{Lemma 2.3.}

(i) If $x$ does not appear free in $P$, and $x: T \triangleright P$ prop then $P$ prop

(ii) If $x$ does not appear free in $t$, and $x: T_{0} \triangleright t: T_{1}$ then $t: T_{1}$

(iii) If $t: T_{1}$ and $x$ does not appear free in $t$ then $x: T_{0} \triangleright t: T_{1}$

(iv) If $P$ prop and $x$ does not appear free in $P$ then $x: T \triangleright P$ prop

Typing of terms is unique in $\mathrm{Z}_{C}$.

Lemma 2.4. If $t: T_{0}$ and $t: T_{1}$ then $T_{0}=T_{1}$

Proof. By the generation lemma (lemma 2.2). 


\section{Lemma 2.5.}

(i) If $P[z / t]$ prop and $t: T$ then $z: T \triangleright P$ prop

(ii) If $z: T \triangleright P$ prop and $t: T$ then $P[z / t]$ prop

(iii) If $t_{1}\left[z / t_{0}\right]: T_{1}$ and $t_{0}: T_{0}$ then $z: T_{0} \triangleright t_{1}: T_{1}$

(iv) If $z: T_{0} \triangleright t_{1}: T_{1}$ and $t_{0}: T_{0}$ then $t_{1}\left[z / t_{0}\right]: T_{1}$

Proof. The pairs (i) and (iii); (ii) and (iv) are each proved by simultaneous induction on the structure of the relevant derivations.

\subsection{The logic of $Z_{C}$}

The proto-judgements of the logic have the form:

$$
\Gamma \vdash_{C} P
$$

where a proto-context $\Gamma$ has the form $\Gamma^{-} ; \Gamma^{+} . \Gamma^{-}$is a type assignment context (a context for the type system) and $\Gamma^{+}$is a set of formulae. These are well-formed according to the following rules.

$$
\overline{\Gamma^{-} \text {context }} \quad \frac{\Gamma^{-} \triangleright P \text { prop } \Gamma \text { context }}{\Gamma^{-} ; P, \Gamma^{+} \text {context }}
$$

Proofs introduce new putative contexts, propositions and terms and the rules must be guarded in some cases by type judgements to ensure they remain type consistent. We shall establish that these conditions of well-formedness are maintained by the rules below (proposition 2.9). For notational simplicity we shall, in the sequel, often write $t^{T}$ for a term $t$ such that $t: T$ in the context in which it appears. For example, $\Gamma \vdash P\left(t^{T}\right)$ implies that, additionally, $\Gamma^{-} \triangleright t: T$.

\section{Definition 2.6.}

$$
t_{0} \equiv t_{1}=_{d f} \forall z \in t_{0} \bullet z \in t_{1} \wedge \forall z \in t_{1} \bullet z \in t_{0}
$$

Definition 2.7. (Logic of $Z_{C}$ ). As before, we omit all data which remain unchanged by a rule.

$$
\begin{aligned}
& \frac{\Gamma \vdash P_{0} \quad \Gamma^{-} \triangleright P_{1} \text { prop }}{\Gamma \vdash P_{0} \vee P_{1}}\left(\vee_{0}^{+}\right) \quad \frac{\Gamma \vdash P_{1} \quad \Gamma^{-} \triangleright P_{0} \text { prop }}{\Gamma \vdash P_{0} \vee P_{1}}\left(\vee_{\mathrm{I}}^{+}\right) \\
& \frac{P_{0} \vee P_{1} \quad P_{0} \vdash P_{2} \quad P_{1} \vdash P_{2}}{P_{2}}\left(\vee^{-}\right) \\
& \frac{\Gamma, P \vdash \perp \quad \Gamma^{-} \triangleright P \text { prop }}{\Gamma \vdash \neg P}\left(\neg^{+}\right) \quad \frac{\neg \neg P}{P}\left(\neg^{-}\right) \quad \frac{P \quad \neg P}{\perp}\left(\perp^{+}\right) \\
& \frac{\Gamma \vdash \perp \Gamma^{-} \triangleright P \text { prop }}{\Gamma \vdash P}\left(\perp^{-}\right) \\
& \frac{\Gamma \vdash P[z / t] \quad \Gamma^{-} \triangleright t: T}{\Gamma \vdash \exists z: T \bullet P}\left(\exists^{+}\right) \\
& \frac{\exists z: T \bullet P_{0} \quad y: T ; P_{0}[z / y] \vdash P_{1}}{P_{1}}\left(\exists^{-}\right) \text {, for fresh } y
\end{aligned}
$$




$$
\begin{aligned}
& \frac{\Gamma, P \text { context }}{\Gamma, P \vdash P} \text { (ass) } \quad \frac{\Gamma^{-} \triangleright t: T}{\Gamma \vdash t=t} \text { (ref) } \\
& \frac{\Gamma^{-} \triangleright \downarrow \cdots l_{i} \Rightarrow t_{i} \cdots \downarrow: T}{\Gamma \vdash\left\langle\cdots l_{i} \Rightarrow t_{i} \cdots \mid\right\rangle \cdot l_{i}=t_{i}}\left(\Rightarrow_{0}^{=}\right) \quad \frac{\Gamma^{-} \triangleright t:\left[\cdots l_{i}: T_{i} \cdots\right]}{\left.\Gamma \vdash \downarrow \cdots l_{i} \Rightarrow t \cdot l_{i} \cdots\right\rangle=t}\left(\Rightarrow_{\mathrm{I}}^{=}\right) \\
& \frac{\Gamma^{-} \triangleright\left(t_{1}, t_{2}\right): T}{\Gamma \vdash\left(t_{1}, t_{2}\right) \cdot 1=t_{1}}\left(()_{\mathrm{O}}^{=}\right) \quad \frac{\Gamma^{-} \triangleright\left(t_{1}, t_{2}\right): T}{\Gamma \vdash\left(t_{1}, t_{2}\right) \cdot 2=t_{2}}\left(()_{\mathrm{I}}^{=}\right) \quad \frac{\Gamma^{-} \vdash t: T_{1} \times T_{2}}{\Gamma \vdash(t .1, t .2)=t}\left(()_{2}^{=}\right) \\
& \frac{t_{0}=t_{1} \quad P\left[z / t_{0}\right]}{P\left[z / t_{1}\right]}(\text { sub }) \quad \frac{P[n / 0] \quad n: \mathbb{N} ; P \vdash P[n / \text { succ } n]}{n: \mathbb{N} \vdash P}\left(\mathbb{N}^{-}\right) \text {, for fresh } n \\
& \frac{\Gamma \vdash P[z / t] \quad \Gamma^{-} \triangleright t: T}{\Gamma \vdash t \in\{z: T \mid P\}}\left(\{\}^{+}\right) \quad \frac{t \in\{z: T \mid P\}}{P[z / t]}\left(\{\}^{-}\right) \\
& \frac{t_{0} \equiv t_{1}}{t_{0}=t_{1}}(\text { ext }) \quad \frac{\Gamma \vdash t . l_{i}=t_{i} \quad \Gamma^{-} \triangleright t: T \quad\left[\cdots l_{i}: T_{i} \cdots\right] \preceq T}{\Gamma \vdash\left(t\left\lceil\left[\cdots l_{i}: T_{i} \cdots\right]\right) . l_{i}=t_{i}\right.}\left(\uparrow^{=}\right)
\end{aligned}
$$

Since contexts are sets we do not require structural rules. The following weakening rule is clearly admissible and may be usefully added:

$$
\frac{\Gamma_{0} \vdash P \quad \Gamma_{0}^{-}, \Gamma_{1}^{-} ; \Gamma_{0}^{+}, \Gamma_{1}^{+} \text {context }}{\Gamma_{0}^{-}, \Gamma_{1}^{-} ; \Gamma_{0}^{+}, \Gamma_{1}^{+} \vdash P}
$$

\subsection{Consequences of the logic for $Z_{C}$}

There should also be a number of equality congruence rules for the terms of $\mathrm{Z}_{C}$. These are not included in the system because they are all easily derivable; essentially because we have the rule $(s u b)$. The following are derived rules of $Z_{C}$ :

$$
\begin{aligned}
& \frac{t_{1}=t_{0}}{t_{0}=t_{1}}(\mathrm{sym}) \quad \frac{t_{0}=t_{1} t_{1}=t_{2}}{t_{0}=t_{2}}(\text { trans }) \quad \frac{t_{0}=t_{2} \quad t_{1}=t_{3}}{\left(t_{0}, t_{1}\right)=\left(t_{2}, t_{3}\right)}\left(={ }_{(}\right) \\
& \frac{\Gamma \vdash t_{0}=t_{1} \quad \Gamma^{-} \triangleright t_{0}: T_{1} \times T_{2}}{\Gamma \vdash t_{0} .1=t_{1} .1}\left(={ }_{. \mathrm{I}}\right) \quad \frac{\Gamma \vdash t_{0}=t_{1} \quad \Gamma^{-} \triangleright t_{0}: T_{1} \times T_{2}}{\Gamma \vdash t_{0} .2=t_{1} .2}\left(={ }_{.2}\right) \\
& \frac{\cdots \quad t_{0 i}=t_{1 i} \cdots}{\downarrow \cdots l_{i} \Rightarrow t_{0 i} \cdots \downarrow=\downarrow \cdots l_{i} \Rightarrow t_{1 i} \cdots \downarrow}(=\Rightarrow) \\
& \frac{\Gamma \vdash t_{0}=t_{1} \quad \Gamma^{-} \triangleright t_{0}:\left[\cdots l_{i}: T_{i} \cdots\right]}{\Gamma \vdash t_{0} . l_{i}=t_{1} . l_{i}}(=. l) \\
& \frac{\Gamma \vdash t_{0}=t_{1} \quad \Gamma^{-} \triangleright t_{0}: T_{1} \quad T_{0} \preceq T_{1}}{\Gamma \vdash t_{0} \uparrow T_{0}=t_{1} \uparrow T_{0}}(=\text { ) }
\end{aligned}
$$

We shall need the following definition, which extends filtering from terms to sets.

Definition 2.8. Let $T_{0} \preceq T_{1}$. Let $z$ and $x$ be fresh variables.

$$
C^{\mathbb{P} T_{1}} \uparrow T_{0}={ }_{d f}\left\{z: T_{0} \mid \exists x: T_{1} \bullet x \in C \wedge z=x \uparrow T_{0}\right\}
$$

Then we have:

$$
\frac{C: \mathbb{P} T_{1} \quad T_{0} \preceq T_{1}}{C \uparrow T_{0}: \mathbb{P} T_{0}}\left(C_{\mathbb{P} \uparrow}\right)
$$


Then we have rules relating filtered terms and filtered sets:

$$
\frac{\Gamma \vdash t \in C \quad \Gamma^{-} \triangleright t: T_{1} \quad T_{0} \preceq T_{1}}{\Gamma \vdash t \uparrow T_{0} \in C \uparrow T_{0}}\left(\in_{\uparrow}^{+}\right)
$$

This follows by rules $\left(C_{\uparrow}\right),\left(\{\}^{+}\right)$and $\left(\exists^{+}\right)$.

$$
\frac{\Gamma \vdash t \in C^{\mathbb{P} T_{0}} \uparrow T \quad \Gamma^{-}, x: T_{0} ; \Gamma^{+}, x \in C, t=x \uparrow T \vdash P}{\Gamma \vdash P}\left(\in_{\uparrow}^{-}\right)
$$

for fresh $x$. This follows by rules $\left(\{\}^{-}\right)$and $\left(\exists^{-}\right)$.

\subsection{Syntactic consistency for $Z_{C}$}

The logic should only enable us to deduce well-formed propositions from wellformed assumptions. This is the content of the next result.

Proposition 2.9. (Syntactic consistency). If $\Gamma \vdash_{C} P$ when $\Gamma$ context then $\Gamma^{-} \triangleright_{C} P$ prop

Proof. By induction on the structure of the derivation $\Gamma \vdash P$. We give one case to illustrate. Suppose that $\Gamma$ context.

Case rule $\left(\exists^{-}\right)$: we may assume ex hypothesi (first premise) that $\Gamma^{-} \triangleright \exists z: T \bullet$ $P_{0}$ prop since we have $\Gamma$ context by assumption. From the generation lemma it follows that $\Gamma^{-}, z: T \triangleright P_{0}$ prop and this, together with the assumption that $\Gamma$ context, is sufficient to show that $\Gamma^{-}, z: T ; \Gamma^{+}, P_{0}$ context. Since we know that the variable $y$ is not free in $P_{0}$ we have, by alpha-conversion (in the meta-language), $\Gamma^{-}, y: T ; \Gamma^{+}, P_{0}[z / y]$ context and then, ex hypothesi (second premise), that $\Gamma^{-}, y: T \triangleright P_{1}$ prop. But since $y$ is not free in $P_{1}$ this reduces to to $\Gamma^{-} \triangleright P_{1}$ prop by lemma $2.3(i)$.

The specification logic $Z_{C}$ is essentially a typed set theory in which, in particular, we have schema types. There are, however, no schemas in $Z_{C}$ and this may seem rather odd since these are archetypical of $Z$. In fact, given the schema types, schemas are just special cases of the comprehensions. Specifically, we may introduce schemas by meta-notational convention using the following definition:

$$
[D \mid P]={ }_{d f}\{z:[D] \mid P[\alpha[D] / z . \alpha[D]]\}
$$

where $z$ is a fresh variable. Note that this device requires us to allow the metavariable $P$ to range over the proto-propositions extended with labels as terms. The right-hand-side is, of course, (proto-)syntactically valid in $Z_{C}$. Given this definition we may provide the following versions of the comprehension rules using the schema notation:

$$
\begin{gathered}
{\left[\frac{\Gamma \vdash P[\alpha[D] / t . \alpha[D]] \quad \Gamma^{-} \triangleright t:[D]}{\Gamma \vdash t \in[D \mid P]}\right]} \\
\frac{t \in[D \mid P]}{P[\alpha[D] / t . \alpha[D]]}
\end{gathered}
$$

It is immediate that equality for schemas is also extensional (see definition 2.6). We shall return to this in far more detail in Section 5.2, where we show how an algebra of schemas can be represented in $Z_{C}$. 
We are proposing that $Z_{C}$ be taken as an adequate base theory within which the much higher-level features of $\mathrm{Z}$ can be interpreted. As such it plays an intermediate role between $Z$ and classical, extensional set theory (which is the intended model for $Z$ ). To show that $Z_{C}$ can play this role we must show that it can be faithfully interpreted in $\mathrm{ZF}$, and we devote the next section to that task. Sections 4 and 5 are then devoted to showing that $Z_{C}$ is adequate for the interpretation of $\mathrm{Z}$.

\section{A model of $Z_{C}$ in $\mathbf{Z F}$}

In this section we provide an interpretation $\llbracket-\rrbracket_{C}$ from the language of $Z_{C}$ into $\mathrm{ZF}$ and present a variety of results. We shall omit the subscript on the interpretation function unless this is essential. The semantics is extremely simple. The novelty lies in our interpretation of schema types as dependent products over a family of sets from a small (in ordinal terms) cumulative universe.

\subsection{Types}

The language of types $Z_{C}$ is given by a simple context free grammar. Such a grammar is understood mathematically to be an inductive definition over an operator which determines a set (the language of the grammar). The closure ordinal for this induction (the ordinal at which iteration of the operator reaches a fixpoint) is $\omega$ because the operator in question is continuous. In other words, the non-terminal operators may be applied finitely, but unboundedly, often. Consequently the type structures which can be described are, from a set-theoretic perspective, rather trivial. Consider, therefore, the following definition in $\mathrm{ZF}$ which constructs a tiny cumulative hierarchy.

(i) $F(0)=\mathbb{N}$

(ii) $F(\alpha+1)=F(\alpha) \cup \mathbb{P} F(\alpha)$

(iii) $F(\omega)=\bigcup_{\alpha<\omega} F(\alpha)$

This function is guaranteed to exist by transfinite induction (in fact only transfinite induction below $\omega .2$ is required) and we then take $F(\omega+1)$ to be the universe within which the type system of $Z_{C}$ may be interpreted. This universe is a set. Let $B$ be an $I$-indexed family of sets over $F(\omega)$. (That is, $B \in I \rightarrow F(\omega+1)$.) Then we can define a dependent function space which is suitable for our purposes as follows:

$$
\Pi_{(X \in I)} \cdot B(X)=_{d f}\{f \in I \rightarrow F(\omega) \mid(\forall i \in I)(f(i) \in B(i))\}
$$

This we can harness to interpret the types of $\mathrm{Z}_{C}$ :

$$
\begin{array}{llll}
\text { (i) } & \llbracket \mathbb{N} \rrbracket & { }_{d f} & \mathscr{N} \\
\text { (ii ) } & \llbracket T_{0} \times T_{1} \rrbracket & { }_{d f} & \llbracket T_{0} \rrbracket \mathscr{X} \llbracket T_{1} \rrbracket \\
\text { (iii) } & \llbracket \mathbb{P} T \rrbracket & ={ }_{d f} & \mathscr{P} \llbracket T \rrbracket \\
\text { (iv) } & \llbracket\left[\cdots l_{i}: T_{i} \cdots\right] \rrbracket & ={ }_{d f} & \Pi_{(X \in I)} . B(X)
\end{array}
$$

where $I=_{d f}\left\{\cdots l_{i} \cdots\right\}$ and $B\left(l_{i}\right)=_{d f} \llbracket T_{i} \rrbracket$. The labels $l_{i}$ can be modelled in ZF in any number of ways, for example as finite ordinals. The only important point is that they be distinguishable from one another. We shall write them in $Z F$ as we do in $\mathrm{Z}_{C}$ for simplicity. 


\subsection{Sets, logic and terms}

We shall translate the entire proto-syntax of $Z_{C}$ into $\mathrm{ZF}$. We begin with the formulae.

$$
\begin{array}{lll}
\llbracket \perp \rrbracket & =_{d f} & (\forall x)(\neg x=x) \\
\llbracket t_{0}=t_{1} \rrbracket & =_{d f} & \llbracket t_{0} \rrbracket=\llbracket t_{1} \rrbracket \\
\llbracket t_{0} \in t_{1} \rrbracket & =_{d f} & \llbracket t_{0} \rrbracket \in \llbracket t_{1} \rrbracket \\
\llbracket \neg P \rrbracket & =_{d f} & \neg \llbracket P \rrbracket \\
\llbracket P_{0} \vee P_{1} \rrbracket & =_{d f} & \llbracket P_{0} \rrbracket \vee \llbracket P_{1} \rrbracket \\
\llbracket \exists z: T \bullet P \rrbracket & =_{d f} & (\exists z)(z \in \llbracket T \rrbracket \wedge \llbracket P \rrbracket)
\end{array}
$$

There are no special conditions to impose with respect to the judgement of propositionhood of $Z_{C}$, since $\mathrm{ZF}$ is an untyped language of sets. As a consequence we may interpret the judgement forms $\Gamma \triangleright_{C} P$ prop to mean that $\llbracket P \rrbracket$ is a wellformed formula of ZF. Since this is true for any $P$, the judgement is (semantically) redundant.

The terms are straightforwardly interpreted. We take the usual definition of cartesian product in ZF in which ordered pairs are defined by $\langle x, y\rangle=_{d f}$ $\{\{x\},\{x, y\}\}$. Then we make use of the maps fst $\in A \mathscr{X} B \rightarrow A$ such that $\langle a, b\rangle \stackrel{f s t}{\mapsto} a$ and snd $\in A \mathscr{X} B \rightarrow B$ such that $\langle a, b\rangle \stackrel{\text { snd }}{\mapsto} b$.

In what follows let $f_{0} \in \llbracket\left[\cdots l_{i}: T_{i} \cdots\right] \rrbracket$ and $f_{0}\left(l_{i}\right)=\llbracket t_{i} \rrbracket$ and $f_{1} \in \llbracket[D] \rrbracket$ and $f_{1}(l)=\llbracket t \rrbracket(l)$ when $l \in \alpha[D]$.

$$
\begin{aligned}
& \llbracket x \rrbracket \quad=d f \quad x \\
& \llbracket n \rrbracket \quad=_{d f} \quad n \\
& \llbracket\{x: T \mid P\} \rrbracket \quad=_{d f} \quad\{\llbracket x \rrbracket \in \llbracket T \rrbracket \mid \llbracket P \rrbracket\} \\
& \llbracket t . l \rrbracket \quad=_{d f} \quad \llbracket t \rrbracket(l) \\
& \llbracket\left\langle\cdots l_{i} \Rightarrow t_{i} \cdots \nabla \rrbracket \quad=_{d f} \quad f_{0}\right. \\
& \llbracket t .1 \rrbracket \quad=d f \quad f s t \llbracket t \rrbracket \\
& \llbracket t .2 \rrbracket \quad=d f \quad \text { snd } \llbracket t \rrbracket \\
& \llbracket\left(t_{0}, t_{1}\right) \rrbracket \quad=d f \quad\left\{\left\{\llbracket t_{0} \rrbracket\right\},\left\{\llbracket t_{0} \rrbracket, \llbracket t_{1} \rrbracket\right\}\right\} \\
& \llbracket t \uparrow[D] \rrbracket \quad=d f \quad f_{1}
\end{aligned}
$$

\subsection{Mathematical results}

As a result of careful design the two crucial semantic results for $Z_{C}$ are easy to prove.

Proposition 3.1. (Soundness of type assignment for $\mathrm{Z}_{C}$ ). If $\Gamma \triangleright_{C} t: T$ then $\llbracket \Gamma \rrbracket \vdash_{Z F} \llbracket t \rrbracket \in \llbracket T \rrbracket$.

Proposition 3.2. (Soundness of $Z_{C}$ logic). If $\Gamma \vdash_{C} P$ then $\llbracket \Gamma \rrbracket \vdash_{Z F} \llbracket P \rrbracket$.

\section{Introducing $\mathrm{Z}$}

The specification logic $Z$ which we introduce in this section will seem a somewhat impoverished version of the $\mathrm{Z}$ one routinely finds in the literature. Our intention is to provide a basic, high-level extension of $Z_{C}$ which itself may be extended, 
by further internal definition, in a variety of ways. It does not seem sensible to us that $\mathrm{Z}$ should aim to provide every feature for every conceivable application; particularly when these may be expressed very simply as notational conventions. What we focus on here will be generalisations in which sets may occur in what are type contexts in $Z_{C}$, and on the basic operations of the schema calculus.

There remain, nonetheless, a number of particularly important notions that it would be a mistake to leave unexamined so, having provided an interpretation for the specification logic of this section, we shall, in the companion paper [HeR99], go on to give an explanation of the most important derived constructs of $\mathrm{Z}$.

\subsection{The language of $Z$}

We first give the proto-syntax for the language of $\mathrm{Z}$ which we consider in this paper. Essentially $Z$ extends $Z_{C}$ by allowing more general forms of propositions, more general forms of sets, and a number of new forms of terms. We shall use the same names for the syntactic categories as we used for $Z_{C}$, except for the declarations, since the $Z_{C}$ category appears as well. In what follows we will always write $D_{C}$ for the category of $Z_{C}$ declarations, permitting us to reuse the category name $D$ for the more general $\mathrm{Z}$ declarations.

Types are as they were in $\mathrm{Z}_{C}$.

$$
T::=\mathbb{N}|\mathbb{P} T| T \times T \mid\left[D_{C}\right]
$$

The proto-syntax for declarations in $\mathrm{Z}$ is, then:

$$
D::=l \in C \quad \mid \quad l \in C ; D
$$

The proto-syntax of propositions:

$$
P::=\perp|t=t| t \in C|\neg P| P \vee P \mid \exists z \in C \bullet P
$$

Finally, we have the proto-syntax for terms. In addition to our use of $C$ as a meta-variable for terms of type $\mathbb{P} T$ we now reserve the meta-variable $S$ for sets of type $\mathbb{P} T$ where $T$ is a schema type. Firstly, then, we have the terms which are sets:

$$
t::=\{z \in C \mid P\}|\mathbb{P} C| C \times C|\mathbb{N}|[D] \mid \lambda z \in C \bullet t
$$

In $\mathrm{Z}$, then, the types appear as a sub-category, or, more precisely, the carrier sets of the types do. These can be formally isolated by means of the following category definitions:

$$
\begin{aligned}
& D^{*}::=l \in T^{*} \mid l \in T^{*} ; D^{*} \\
& T^{*}::=\mathbb{N}\left|T^{*} \times T^{*}\right| \mathbb{P} T^{*} \mid\left[D^{*}\right]
\end{aligned}
$$

Since $D^{*}$ is just the $\mathrm{Z}$ image of the $\mathrm{Z}_{C}$ declarations, we can take all the operations defined over $D_{C}$ as inducing similar operations over $D^{*}$.

We have established a notational shift from conventional presentations of $\mathrm{Z}$ but, we feel, it is justified. Most particularly, allowing $t: C$ would suggest that we are permitting sets to be types, which we are not: such an approach would make typechecking undecidable. Writing $t \in T^{*}$ on the other hand suggests that we are permitting types to be sets which is precisely what $\mathrm{Z}$ allows. Additionally, the colon is a judgement of the type assignment and propositionhood system and this never assigns anything other than a type (a name) to a term. Consequently, 
it would be somewhat confusing to permit more general usage for the type assignment symbol elsewhere in the language. These scruples arise here because we are dealing with logical systems and not simply the language. Perhaps we are being too fastidious, but the strict distinction between a type and its carrier is well recognised (e.g. [Spi92] p. 24). What might be a reasonable abuse of language (writing $T$ in place of the carrier set $T^{*}$ ), we feel, may be more easily accommodated than an abuse of logic (writing $t: T$ in place of $t \in T^{*}$ (or $t \in T)$ ). Indeed we will not have to burden the presentation by distinguishing a type and its carrier precisely because we will insist on the correct logical relation in type judgements and in membership propositions.

Among the set comprehensions we shall, as before, isolate the schemas as a special case with the special meta-notation (where $z$ is fresh):

$$
[D \mid P]=_{d f}\{z \in[D] \mid P[\alpha[D] / z . \alpha[D]]\}
$$

We then extend the term language with schema expressions:

$$
t::=\cdots|[D \mid P]| S \vee S|S \backslash[l: T]| \neg S \mid S\left[l_{1} \leftarrow l_{0}\right]
$$

Note that all such expressions are included as sets in Z. We only use the unusual notation for renaming in order to prevent confusion with substitution in the meta-language. Schema hiding is, in standard approaches, equivalent to schema existential quantification (e.g. [WoD96] p. 181).

Disjunction, hiding, negation and renaming are sufficient to permit definitions for conjunction, implication, equivalence, pre-condition, composition and piping to be constructed using the usual definitions. We shall have more to say about this in the companion paper [HeR99].

The proto-syntax of terms is completed by means of the following:

$$
\begin{aligned}
t::= & \cdots|x| n|t . l| \downarrow \cdots l \Rightarrow t \cdots \downarrow|t .1| t .2|(t, t)| \\
& t \uparrow[D] \mid \text { let } x==t \text { in } t \mid(\lambda z \in C \bullet t) t
\end{aligned}
$$

We shall write $t_{0} \mapsto t_{1}$ as a synonym for $\left(t_{0}, t_{1}\right)$ when the pair is considered as a maplet, that is, as an element of a function.

\subsection{Type assignment and propositionhood in $\mathrm{Z}$}

Definition 4.1. The judgements of the system again have the following forms:

$$
\begin{aligned}
& \Gamma \triangleright_{Z} P \text { prop } \\
& \Gamma \triangleright_{Z} t: T
\end{aligned}
$$

The contexts, as usual, are partial functions giving type assignments for variables. As before, in giving the rules, we will omit any data which are not changed by a rule.

$$
\begin{aligned}
& \overline{\perp \text { prop }}\left(Z_{\perp}\right) \quad \frac{t_{0}: T t_{1}: T}{t_{0}=t_{1} \text { prop }}\left(Z_{=}\right) \quad \frac{t: T \quad C: \mathbb{P} T}{t \in C \text { prop }}\left(Z_{\in}\right) \\
& \frac{P \text { prop }}{\neg P \text { prop }}\left(Z_{\neg}\right) \quad \frac{P_{0} \text { prop } P_{1} \text { prop }}{P_{0} \vee P_{1} \text { prop }}\left(Z_{\vee}\right) \quad \frac{C: \mathbb{P} T z: T \triangleright P \text { prop }}{\exists z \in C \bullet P \text { prop }} \\
& \overline{x: T \triangleright x: T}\left(Z_{x}\right) \quad \overline{0: \mathbb{N}}\left(Z_{\circ}\right) \quad \frac{n: \mathbb{N}}{\text { succ } n: \mathbb{N}}\left(Z_{s}\right)
\end{aligned}
$$




$$
\begin{aligned}
& \frac{S_{0}: \mathbb{P} T_{0} \quad S_{1}: \mathbb{P} T_{1}}{S_{0} \vee S_{1}: \mathbb{P}\left(T_{0} \vee T_{1}\right)}\left(Z_{\vee S}\right) \quad \frac{S: \mathbb{P} T}{S \backslash\left[l: T_{1}\right]: \mathbb{P}\left(T \backslash\left[l: T_{1}\right]\right)}\left(Z_{h}\right) \\
& \frac{S: T}{S\left[l_{0} \leftarrow l_{1}\right]: T\left[l_{0} \leftarrow l_{1}\right]}\left(Z_{r}\right), l_{1} \notin \alpha T \\
& \frac{C: \mathbb{P} T \quad z: T \triangleright P \text { prop }}{\{z \in C \mid P\}: \mathbb{P} T}\left(Z_{\{\}}\right) \quad \frac{S: T}{\neg S: T}\left(Z_{\neg S}\right) \quad \frac{C: \mathbb{P} T}{\mathbb{P} C: \mathbb{P} \mathbb{P} T}\left(Z_{\mathbb{P}}\right) \\
& \frac{C_{0}: \mathbb{P} T_{0} \quad C_{1}: \mathbb{P} T_{1}}{C_{0} \times C_{1}: \mathbb{P}\left(T_{0} \times T_{1}\right)}\left(Z_{\times}\right) \quad \overline{\mathbb{N}: \mathbb{P N}}\left(Z_{\mathbb{N}}\right) \\
& \frac{\cdots \quad C: \mathbb{P} T \cdots}{[\cdots l \in C \cdots]: \mathbb{P}[\cdots l: T \cdots]}\left(Z_{[]}\right) \quad \frac{t:\left[\cdots l_{i}: T \cdots\right]}{t . l_{i}: T}(Z .) \\
& \frac{C: \mathbb{P} T_{0} \quad z: T_{0} \triangleright t: T_{1}}{\lambda z \in C \bullet t: \mathbb{P}\left(T_{0} \times T_{1}\right)}\left(Z_{\lambda}\right) \quad \frac{\lambda z \in C \bullet t_{1}: \mathbb{P}\left(T_{0} \times T_{1}\right) \quad t_{0}: T_{0}}{\left(\lambda z \in C \bullet t_{1}\right) t_{0}: T_{1}}\left(Z_{a p}\right) \\
& \frac{\cdots \quad t_{i}: T_{i} \quad \cdots}{\left\langle\cdots l_{i} \Rightarrow t_{i} \cdots\right\rangle:\left[\cdots l_{i}: T_{i} \cdots\right]}\left(Z_{\Rightarrow}\right) \\
& \frac{t: T_{1} \times T_{2}}{t .1: T_{1}}\left(Z_{\mathrm{I}}\right) \quad \frac{t: T_{1} \times T_{2}}{t .2: T_{2}}\left(Z_{2}\right) \\
& \frac{t_{0}: T_{0} \quad t_{1}: T_{1}}{\left(t_{0}, t_{1}\right): T_{0} \times T_{1}}\left(Z_{()}\right) \quad \frac{t_{0}: T_{0} \quad x: T_{0} \triangleright t_{1}: T_{1}}{\text { let } x==t_{0} \text { in } t_{1}: T_{1}}\left(Z_{\text {let }}\right) \\
& \frac{t:\left[D_{1}\right] \quad\left[D_{0}\right] \preceq\left[D_{1}\right]}{t \uparrow\left[D_{0}\right]:\left[D_{0}\right]}\left(Z_{\uparrow}\right)
\end{aligned}
$$

Similar results to those we exhibited for the corresponding system for $Z_{C}$ hold for the system for $Z$.

Lemma 4.2. The generation lemma for $Z$ holds.

\section{Lemma 4.3.}

(i) If $x$ does not appear free in $P$, and $x: T \triangleright P$ prop then $P$ prop

(ii) If $x$ does not appear free in $t$, and $x: T_{0} \triangleright t: T_{1}$ then $t: T_{1}$

(iii) If $t: T_{1}$ and $x$ does not appear free in $t$ then $x: T_{0} \triangleright t: T_{1}$

(iv) If $P$ prop and $x$ does not appear free in $P$ then $x: T \triangleright P$ prop

Typing of terms is also unique in $\mathrm{Z}$.

Lemma 4.4. If $t: T_{0}$ and $t: T_{1}$ then $T_{0}=T_{1}$

Proof. By the generation lemma (lemma 4.2).

\section{Lemma 4.5.}

(i) If $P[z / t]$ prop and $t: T$ then $z: T \triangleright P$ prop

(ii) If $z: T \triangleright P$ prop and $t: T$ then $P[z / t]$ prop

(iii) If $t_{1}\left[z / t_{0}\right]: T_{1}$ and $t_{0}: T_{0}$ then $z: T_{0} \triangleright t_{1}: T_{1}$

(iv) If $z: T_{0} \triangleright t_{1}: T_{1}$ and $t_{0}: T_{0}$ then $t_{1}\left[z / t_{0}\right]: T_{1}$

Lemma 4.6. $\triangleright T: \mathbb{P} T$ for all types $T$.

Proof. An easy induction on the structure of types. 
Lemma 4.7. The following rule is derivable:

$$
\frac{C: \mathbb{P} T_{1} \quad T_{0} \preceq T_{1}}{C \uparrow T_{0}: \mathbb{P} T_{0}}\left(Z_{\mathbb{P} \uparrow}\right)
$$

\subsection{A logic for $Z$}

Definition 4.8. (Logic of $\mathbf{Z}$ ). The judgements of the logic have the form:

$$
\Gamma \vdash_{Z} P
$$

Contexts $\Gamma$ have the form $\Gamma^{-} ; \Gamma^{+}$as they did in Section 2.4, and these are well-formed by means of analogous rules introduced earlier for $Z_{C}$.

$$
\begin{aligned}
& \frac{\Gamma \vdash P_{0} \quad \Gamma^{-} \triangleright P_{1} \text { prop }}{\Gamma \vdash P_{0} \vee P_{1}}\left(\vee_{0}^{+}\right) \quad \frac{\Gamma \vdash P_{1} \Gamma^{-} \triangleright P_{0} \text { prop }}{\Gamma \vdash P_{0} \vee P_{1}}\left(\vee_{\mathrm{I}}^{+}\right) \\
& \frac{P_{0} \vee P_{1} \quad P_{0} \vdash P_{2} \quad P_{1} \vdash P_{2}}{P_{2}}\left(\vee^{-}\right) \\
& \frac{\Gamma, P \vdash \perp \quad \Gamma^{-} \triangleright P \text { prop }}{\Gamma \vdash \neg P}\left(\neg^{+}\right) \quad \frac{\neg \neg P}{P}\left(\neg^{-}\right) \quad \frac{P \quad \neg P}{\perp}\left(\perp^{+}\right) \\
& \frac{\Gamma \vdash \perp \Gamma^{-} \triangleright P \text { prop }}{\Gamma \vdash P}\left(\perp^{-}\right) \quad \frac{P[z / t] t \in C}{\exists z \in C \bullet P}\left(\exists^{+}\right) \\
& \frac{\Gamma \vdash \exists z \in C \bullet P_{0} \quad \Gamma^{-} \triangleright C: \mathbb{P} T \quad \Gamma^{-}, y: T ; \Gamma^{+}, y \in C, P_{0}[z / y] \vdash P_{1}}{P_{1}}
\end{aligned}
$$

for fresh $y$.

$$
\begin{aligned}
& \frac{\Gamma, P \text { context }}{\Gamma, P \vdash P} \text { (ass) } \quad \frac{\Gamma^{-} \triangleright t: T}{\Gamma \vdash t=t} \text { (ref) } \\
& \frac{t_{0}=t_{1} \quad P\left[z / t_{0}\right]}{P\left[z / t_{1}\right]}(s u b) \\
& \frac{\Gamma^{-} \triangleright\left\langle\cdots l_{i} \Rightarrow t_{i} \cdots \downarrow: T\right.}{\Gamma \vdash \downarrow \cdots l_{i} \Rightarrow t_{i} \cdots \downarrow l_{i}=t_{i}}\left(\Rightarrow^{=}\right) \quad \frac{\Gamma^{-} \triangleright t:\left[\cdots l_{i}: T_{i} \cdots\right]}{\left.\Gamma \vdash \downarrow \cdots l_{i} \Rightarrow t \cdot l_{i} \cdots\right\rangle=t}\left(\Rightarrow^{=}\right) \\
& \frac{\Gamma^{-} \triangleright\left(t_{1}, t_{2}\right): T}{\Gamma \vdash\left(t_{1}, t_{2}\right) \cdot 1=t_{1}}\left(()_{\mathrm{O}}^{=}\right) \quad \frac{\Gamma^{-} \triangleright\left(t_{1}, t_{2}\right): T}{\Gamma \vdash\left(t_{1}, t_{2}\right) \cdot 2=t_{2}}\left(()_{\mathrm{I}}^{=}\right) \quad \frac{\Gamma^{-} \vdash t: T_{1} \times T_{2}}{\Gamma \vdash(t .1, t .2)=t}\left(()_{2}^{=}\right) \\
& \frac{P[z / t] \quad t \in C}{t \in\{z \in C \mid P\}}\left(\{\}^{+}\right) \quad \frac{t \in\{z \in C \mid P\}}{t \in C}\left(\{\}_{0}^{-}\right) \quad \frac{t \in\{z \in C \mid P\}}{P[z / t]}\left(\{\}_{\mathrm{I}}^{-}\right) \\
& \frac{t \notin S}{t \in \neg S}\left(\neg S^{+}\right) \quad \frac{t \in \neg S}{t \notin S}\left(\neg S^{-}\right) \\
& \frac{t \in S}{t\left[l_{0} \leftarrow l_{1}\right] \in S\left[l_{0} \leftarrow l_{1}\right]}\left(S_{\leftarrow}^{+}\right), l_{1} \notin \alpha S \quad \frac{t \in S\left[l_{0} \leftarrow l_{1}\right]}{t\left[l_{1} \leftarrow l_{0}\right] \in S}\left(S_{\leftarrow}^{-}\right), l_{1} \notin \alpha S \\
& \frac{\Gamma \vdash t \in S \quad \Gamma^{-} \triangleright t: T_{0}}{\Gamma \vdash t \uparrow T_{0} \backslash\left[l: T_{1}\right] \in S \backslash\left[l: T_{1}\right]}\left(S_{h}^{+}\right)
\end{aligned}
$$




$$
\frac{\Gamma \vdash t^{\hat{T}} \in S^{\mathbb{P} T_{0}} \backslash\left[l: T_{1}\right] \quad \Gamma^{-}, y: T_{0} ; \Gamma^{+}, y \in S, y \uparrow \hat{T}=t \vdash P}{\Gamma \vdash P}\left(S_{h}^{-}\right)
$$

where $\hat{T}$ is $T_{0} \backslash\left[l: T_{1}\right]$ and for fresh $y$.

$$
\begin{gathered}
\frac{\Gamma \vdash t \uparrow T_{0} \in S_{0} \quad \Gamma^{-} \triangleright S_{1}: \mathbb{P} T_{1} \quad \Gamma^{-} \triangleright t: T_{0} \vee T_{1}}{\Gamma \vdash t \in S_{0} \vee S_{1}}\left(S_{\vee_{0}}^{+}\right) \\
\frac{\Gamma \vdash t \uparrow T_{1} \in S_{1} \quad \Gamma^{-} \triangleright S_{0}: \mathbb{P} T_{0} \quad \Gamma^{-} \triangleright t: T_{0} \vee T_{1}}{\Gamma \vdash t \in S_{0} \vee S_{1}}\left(S_{\vee_{1}}^{+}\right) \\
\frac{t \in S_{0}^{\mathbb{P} T_{0}} \vee S_{1}^{\mathbb{P} T_{1}} \quad t \uparrow T_{0} \in S_{0} \vdash P \quad t \uparrow T_{1} \in S_{1} \vdash P}{P}\left(S_{\vee}^{-}\right) \\
\frac{\Gamma^{-}, z: T ; \Gamma^{+}, z \in C_{0} \vdash z \in C_{1} \quad \Gamma^{-} \triangleright C_{0}: \mathbb{P} T}{\Gamma \vdash C_{0} \in \mathbb{P} C_{1}}\left(\mathbb{P}^{+}\right) \\
\frac{C_{0} \in \mathbb{P} C_{1} \quad t \in C_{0}}{t \in C_{1}}\left(\mathbb{P}^{-}\right) \\
\frac{t \in C_{1} \times C_{2}}{t .1 \in C_{1}}\left(\times_{\mathrm{I}}^{-}\right) \quad \frac{t \in C_{1} \times C_{2}}{t .2 \in C_{2}}\left(\mathrm{t}_{2} \in C_{2}\right)
\end{gathered}
$$

$\overline{0 \in \mathbb{N}}\left(\mathbb{N}_{\mathrm{o}}^{+}\right) \frac{n \in \mathbb{N}}{\text { succ } n \in \mathbb{N}}\left(\mathbb{N}_{\mathrm{I}}^{+}\right) \frac{P[n / 0] n: \mathbb{N} ; P \vdash P[n / \text { succ } n]}{n: \mathbb{N} \vdash P}\left(\mathbb{N}^{-}\right)$, fresh $n$

$$
\begin{gathered}
\left.\frac{\cdots t_{i} \in C_{i} \quad \cdots}{\left\langle\cdots \cdots l_{i} \Rightarrow t_{i} \cdots\right\rangle \in\left[\cdots l_{i} \in C_{i} \cdots\right]}\left(\square^{+}\right) \quad \frac{t \in\left[\cdots l_{i} \in C_{i} \cdots\right]}{t . l_{i} \in C_{i}}(]^{-}\right) \\
\frac{\Gamma \vdash t_{0} \in C \quad \Gamma^{-} \triangleright C: \mathbb{P} T_{0} \quad \Gamma^{-}, z: T_{0} \triangleright t_{1}: T_{1}}{\Gamma \vdash t_{0} \mapsto t_{1}\left[z / t_{0}\right] \in\left(\lambda z \in C \bullet t_{1}\right)}\left(\lambda^{+}\right) \\
\frac{t_{0} \in\left(\lambda z \in C \bullet t_{1}\right)}{t_{0} .1 \in C}\left(\lambda_{0}^{-}\right) \quad \frac{t_{0} \in\left(\lambda z \in C \bullet t_{1}\right)}{t_{0} \cdot 2=t_{1}\left[z / t_{0} .1\right]}\left(\lambda_{\mathrm{I}}^{-}\right) \\
\frac{\Gamma^{-} \triangleright t_{0}: T_{0} \quad \Gamma^{-}, z: T_{0} \triangleright t_{1}: T_{1} \quad \Gamma \vdash t_{0} \in C}{\Gamma \vdash\left(\lambda z \in C \bullet t_{1}\right) t_{0}=t_{1}\left[z / t_{0}\right]}\left(\lambda^{=}\right) \\
\frac{\Gamma^{-} \triangleright t_{0}: T_{0} \quad \Gamma^{-}, z: T_{0} \triangleright t_{1}: T_{1}}{\Gamma \vdash l e t z==t_{0} \text { in } t_{1}=t_{1}\left[z / t_{0}\right]}(l e t) \\
\frac{\Gamma \vdash t . l_{i}=t_{i} \quad \Gamma^{-} \triangleright t: T \quad\left[\cdots l_{i}: T_{i} \cdots\right] \leq T}{\Gamma \vdash\left(t\left\lceil\left[\cdots l_{i}: T_{i} \cdots\right]\right) l_{i}=t_{i}\right.}
\end{gathered}
$$

\subsection{Consequences of the logic for $Z$}

There are, as was the case with $Z_{C}$, a large number of congruence rules for equality which are all derivable using substitution. 


$$
\begin{aligned}
& \frac{t_{1}=t_{0}}{t_{0}=t_{1}}(\mathrm{sym}) \quad \frac{t_{0}=t_{1} t_{1}=t_{2}}{t_{0}=t_{2}}(\text { trans }) \quad \frac{t_{0}=t_{2} \quad t_{1}=t_{3}}{\left(t_{0}, t_{1}\right)=\left(t_{2}, t_{3}\right)}(=()) \\
& \frac{\Gamma \vdash t_{0}=t_{1} \quad \Gamma^{-} \triangleright t_{0}: T_{1} \times T_{2}}{\Gamma \vdash t_{0} .1=t_{1} .1}\left(=_{. \mathrm{I}}\right) \quad \frac{\Gamma \vdash t_{0}=t_{1} \quad \Gamma^{-} \triangleright t_{0}: T_{1} \times T_{2}}{\Gamma \vdash t_{0} .2=t_{1} .2}\left(={ }_{.2}\right) \\
& \frac{\cdots \quad t_{0 i}=t_{1 i} \cdots}{\left.\left\langle\cdots l_{i} \Rightarrow t_{0 i} \cdots\right\rangle=\downarrow \cdots l_{i} \Rightarrow t_{1 i} \cdots\right\rangle}(=\Rightarrow) \\
& \frac{\Gamma \vdash t_{0}=t_{1} \quad \Gamma^{-} \triangleright t_{0}:\left[\cdots l_{i}: T_{i} \cdots\right]}{\Gamma \vdash t_{0} . l_{i}=t_{1} . l_{i}}(=. l) \\
& \frac{\Gamma \vdash C_{0}=C_{1} \quad \Gamma^{-} \triangleright\left\{z \in C_{0} \mid P\right\}: \mathbb{P} T}{\Gamma \vdash\left\{z \in C_{0} \mid P\right\}=\left\{z \in C_{1} \mid P\right\}}(=\{\}) \quad \frac{C_{0}=C_{1}}{\mathbb{P} C_{0}=\mathbb{P} C_{1}}(=\mathbb{P}) \\
& \frac{C_{0}=C_{2} \quad C_{1}=C_{3}}{C_{0} \times C_{1}=C_{2} \times C_{3}}(=\times) \\
& \frac{S_{0}=S_{2} \quad S_{1}=S_{3}}{S_{0} \vee S_{1}=S_{2} \vee S_{3}}(=\vee) \\
& \frac{S_{0}=S_{1}}{S_{0} \backslash[l: T]=S_{1} \backslash[l: T]}(=\backslash) \quad \frac{S_{0}=S_{1}}{S_{0}\left[l_{0} \leftarrow l_{1}\right]=S_{1}\left[l_{0} \leftarrow l_{1}\right]}\left(={ }_{\leftarrow}\right), l_{1} \notin \alpha S_{0} \\
& \frac{S_{0}=S_{1}}{\neg S_{0}=\neg S_{1}}(=\neg) \\
& \frac{\Gamma \vdash C_{0}=C_{1} \quad \Gamma, z: T \vdash t_{0}=t_{1} \quad \Gamma^{-} \triangleright C_{0}: \mathbb{P} T}{\Gamma \vdash \lambda z \in C_{0} \bullet t_{0}=\lambda z \in C_{1} \bullet t_{1}}\left(=\lambda_{\lambda}\right)
\end{aligned}
$$

for fresh $z$.

$$
\begin{gathered}
\frac{\Gamma \vdash t_{0}=t_{2} \quad \Gamma^{-} \triangleright t_{0}: T \quad \Gamma, x: T \vdash t_{1}=t_{3}}{\Gamma \vdash \text { let } x==t_{0} \text { in } t_{1}=\text { let } x==t_{2} \text { in } t_{3}}\left(={ }_{\text {let }}\right) \\
\frac{\lambda z \in C_{0} \bullet t_{0}=\lambda z \in C_{1} \bullet t_{1} \quad t_{2}=t_{3} \quad t_{2} \in C_{0}}{\left(\lambda z \in C_{0} \bullet t_{0}\right) t_{2}=\left(\lambda z \in C_{1} \bullet t_{1}\right) t_{3}}\left(={ }_{\text {app }}\right) \\
\frac{\Gamma \vdash t_{0}=t_{1} \quad \Gamma^{-} \triangleright t_{0}: T_{1} \quad T_{0} \preceq T_{1}}{\Gamma \vdash t_{0} \uparrow T_{0}=t_{1} \uparrow T_{0}} \quad(=\uparrow)
\end{gathered}
$$

Set equality in $Z$ is, like in $Z_{C}$, extensional. The necessary rules are also part of the logic for $Z$. The rule $\left(\in_{\uparrow}^{+}\right)$, that relates filtered terms and filtered sets in $Z_{C}$ also generalises to $Z$.

Lemma 4.9. The following rule is admissible:

$$
\frac{t: T}{t \in T}
$$

Proof. By induction on the structure of the term $t$. For example:

Case $t .1$ : we may assume that $t .1: T_{1}$ for some type $T_{1}$. By lemma 4.2 we have $t: T_{1} \times T_{2}$ for some type $T_{2}$. Ex hypothesi we then obtain $t \in T_{1} \times T_{2}$ and then $t .1 \in T_{1}$, by rule $\left(\times_{1}^{-}\right)$, as required. 
Schemas were introduced in $\mathrm{Z}$, as in $\mathrm{Z}_{C}$, by convention. This induces immediately the following rules:

$$
\frac{P[\alpha[D] / t . \alpha[D]] \quad t \in[D]}{t \in[D \mid P]}\left(S^{+}\right) \quad \frac{t \in[D \mid P]}{P[\alpha[D] / t . \alpha[D]]}\left(S_{\mathrm{O}}^{-}\right) \quad \frac{t \in[D \mid P]}{t \in[D]}\left(S_{\mathrm{I}}^{-}\right)
$$

It is then possible to prove those relationships which are commonly used to describe (occasionally to define) the schema operators in the literature. This begins the task of establishing an equational logic for $\mathrm{Z}$ which is justified by the logic. The equations all have premises which ensure that the equalities are well-formed.

Lemma 4.10.

$$
\frac{\Gamma^{-} \triangleright\left[D^{*} \mid P\right]: \mathbb{P}\left[D^{*}\right]}{\Gamma \vdash \neg\left[D^{*} \mid P\right]=\left[D^{*} \mid \neg P\right]}\left(\neg^{=}\right)
$$

Proof. Note that the declarations must range over types. It is well known that this equation fails if the declarations range over sets in general. Assume that $\Gamma^{-} \triangleright\left[D^{*} \mid P\right]: \mathbb{P}\left[D^{*}\right]$. This implies that the equation is a proposition and that both sides have the type $\mathbb{P}\left[D^{*}\right]$.

Case $(\subseteq)$ : let $t:\left[D^{*}\right]$. Suppose that $t \in \neg\left[D^{*} \mid P\right]$. Using rule $\left(\neg S^{-}\right)$this is $t \notin\left[D^{*} \mid P\right]$, or equivalently $\neg\left(t \in\left[D^{*}\right] \wedge P\left[\alpha\left[D^{*}\right] / t . \alpha\left[D^{*}\right]\right]\right)$. Using De Morgan's law, this is just $t \notin\left[D^{*}\right] \vee \neg P\left[\alpha\left[D^{*}\right] / t . \alpha\left[D^{*}\right]\right]$. Assume that $t \notin\left[D^{*}\right]$. Using lemma 4.9 we obtain $t \in\left[D^{*}\right]$ from the assumption and hence we conclude that $\perp$, whence, by rule $\left(\perp^{-}\right), \neg P\left[\alpha\left[D^{*}\right] / t \cdot \alpha\left[D^{*}\right]\right]$. This now also follows by rule $\left(\vee^{-}\right)$ from the disjunction above. From this, and $t \in\left[D^{*}\right]$, we finally conclude, by rule $\left(S^{+}\right)$, that $t \in\left[D^{*} \mid \neg P\right]$ as required.

Case (つ): let $t:\left[D^{*}\right]$. Suppose that $t \in\left[D^{*} \mid \neg P\right]$. Using rules $\left(S_{0}^{-}\right)$and $\left(S_{\mathrm{I}}^{-}\right)$we obtain: $\neg P\left[\alpha\left[D^{*}\right] / t . \alpha\left[D^{*}\right]\right]$ and $t \in\left[D^{*}\right]$. By rule $\left(\{\}^{+}\right)$and propositional logic we then conclude that $t \notin\left[D^{*} \mid P\right]$ which, by rule $\left(\neg S^{+}\right)$, is $t \in \neg\left[D^{*} \mid P\right]$ as required.

\section{Lemma 4.11.}

$$
\frac{\Gamma^{-} \triangleright\left[D_{0}^{*} \mid P_{0}\right]: \mathbb{P} T_{0} \quad \Gamma^{-} \triangleright\left[D_{1}^{*} \mid P_{1}\right]: \mathbb{P} T_{1}}{\Gamma \vdash\left[D_{0}^{*} \mid P_{0}\right] \vee\left[D_{1}^{*} \mid P_{1}\right]=\left[D_{0}^{*} \vee D_{1}^{*} \mid P_{0} \vee P_{1}\right]}\left(\vee^{=}\right)
$$

It is important to remember that in our framework labels and variables are distinct, consequently there is no possibility of global variable capture to be addressed here. However in the case of hiding, the distinction between variables and labels leads to a minor notational variation:

Lemma 4.12.

$$
\frac{\Gamma^{-} \triangleright\left[D^{*} \mid P\right]: \mathbb{P} T_{1}}{\Gamma \vdash\left[D^{*} \mid P\right] \backslash[l: T]=\left[D^{*} \backslash[l: T] \mid \exists z: T \bullet P[l / z]\right]}(\backslash)
$$

for fresh $z$.

In addition we have an equation relating general declarations over sets to declarations over types. This, by iteration, enables us to remove all non-type sets from the declarations of $\mathrm{Z}$ schemas in the equational logic. 
Lemma 4.13.

$$
\frac{\Gamma^{-} \triangleright[D ; l \in C \mid P]: \mathbb{P} T_{1} \quad \Gamma^{-} \triangleright C: \mathbb{P} T}{\Gamma \vdash[D ; l \in C \mid P]=[D ; l \in T \mid l \in C \wedge P]}\left(\in{ }^{=}\right)
$$

\subsection{Syntactic consistency for $Z$}

We must, of course, ensure that the rules of the logic are syntactically consistent.

Proposition 4.14. If $\Gamma \vdash_{Z} P$ when $\Gamma$ context then $\Gamma^{-} \triangleright_{Z} P$ prop

Proof. By induction on the structure of the derivation $\Gamma \vdash P$. We give one case for illustration. Suppose that $\Gamma$ context.

Case rule $\left(S_{\vee}^{-}\right)$: We have, immediately, that: $\Gamma^{-} \triangleright S_{0}: \mathbb{P} T_{0}$ and $\Gamma^{-} \triangleright S_{1}: \mathbb{P} T_{1}$. Using lemma 4.2 we have, ex hypothesi from the first premise, that: $\Gamma^{-} \triangleright t$ : $\mathbb{P}\left(T_{0} \vee T_{1}\right)$. Then, because $T_{0} \preceq T_{0} \vee T_{1}$ and $T_{1} \preceq T_{0} \vee T_{1}$, it follows, by rule $\left(Z^{\uparrow}\right)$, that $\Gamma^{-} \triangleright t \uparrow T_{0}: T_{0}$ and $\Gamma^{-} \triangleright t \uparrow T_{1}: T_{1}$. Hence we have, using rule $\left(Z_{\in}\right): \Gamma^{-} \triangleright t \uparrow T_{0} \in S_{0}$ prop and $\Gamma^{-} \triangleright t \uparrow T_{1} \in S_{1}$ prop. This establishes that: $\Gamma, t \uparrow T_{0} \in S_{0}$ context and $\Gamma, t \uparrow T_{1} \in S_{1}$ context. Finally, using just the first of these, we conclude: $\Gamma^{-} \triangleright P$ prop, ex hypothesi from the second premise of the rule.

\section{An interpretation of $\mathrm{Z}$ in $\mathrm{Z}_{C}$}

In this section we describe a translation $\llbracket-\rrbracket_{Z}$ of $Z$, as described above, into $Z_{C}$, our core specification logic. This translation (unlike normalisation processes for $Z$ which are found in the literature) is compositional. This can be achieved because we have made precise the notions of propositionhood and type assignment. Indeed, since well-formed $\mathrm{Z}$ sentences are those which satisfy the rules of definition 4.1, we shall make use, where necessary, of the type information associated with $\mathrm{Z}$ terms. As before, we omit the subscript on the translation function unless it is essential.

\subsection{Propositions}

The language of $\mathrm{Z}$ propositions is only marginally more complicated than that of $Z_{C}$. The translation is, for the main part, transparent:

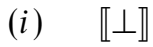
(ii) $\llbracket t_{0}=t_{1} \rrbracket$
$\begin{array}{ll}={ }_{d f} & \perp \\ ={ }_{d f} & \llbracket t_{0} \rrbracket=\llbracket t_{1} \rrbracket\end{array}$
(iii) $\llbracket t \in C \rrbracket$
$={ }_{d f} \quad \llbracket t \rrbracket \in \llbracket C \rrbracket$
(iv) $\llbracket \neg P \rrbracket$
$={ }_{d f} \quad \neg \llbracket P \rrbracket$
(v) $\llbracket P_{0} \vee P_{1} \rrbracket$
$=_{d f} \quad \llbracket P_{0} \rrbracket \vee \llbracket P_{1} \rrbracket$
(vi ) $\llbracket \exists z \in C^{\mathbb{P} T} \bullet P \rrbracket \quad{ }_{d f} \quad \exists z: T \bullet z \in \llbracket C \rrbracket \wedge \llbracket P \rrbracket$ 


\subsection{Schema terms}

The three basic $Z$ schema calculus operations can be translated into $Z_{C}$ as follows. Note that we only interpret type-correct $Z$ and, as a consequence, we may make use of the relevant typing information.

Let $x$ and $z$ be a fresh variables, then:

$$
\begin{aligned}
& \llbracket \neg S^{\mathbb{P} T} \rrbracket \quad=_{d f} \quad\{z: T \mid z \notin \llbracket S \rrbracket\} \\
& \llbracket S_{0}{ }^{\mathbb{P} T_{0}} \vee S_{1}{ }^{\mathbb{P} T_{1}} \rrbracket \quad=_{d f} \quad\left\{z: T_{0} \vee T_{1} \mid z \nmid T_{0} \in \llbracket S_{0} \rrbracket \vee z \nmid T_{1} \in \llbracket S_{1} \rrbracket\right\} \\
& \text { (iii) } \llbracket S^{\mathbb{P} T_{0}} \backslash\left[l: T_{1}\right] \rrbracket \quad=_{d f} \quad\left\{z: T_{0} \backslash\left[l: T_{1}\right] \mid\right. \\
& \left.\exists x: T_{0} \bullet x \in \llbracket S \rrbracket \wedge z=x \uparrow T_{0} \backslash\left[l: T_{1}\right]\right\}
\end{aligned}
$$

\subsection{Set terms}

There are a number of new forms of set available in $\mathrm{Z}$, translated as follows. Again, $z$ is a fresh variable:

(i)

$\llbracket[D \mid P]^{\mathbb{P} T} \rrbracket \quad=d f \quad\{z: T \mid z \in \llbracket[D] \rrbracket \wedge \llbracket P[\alpha T / z . \alpha T] \rrbracket\}$

(ii) $\quad \llbracket\left\{z \in C^{\mathbb{P} T} \mid P\right\} \rrbracket \quad=_{d f} \quad\{z: T \mid z \in \llbracket C \rrbracket \wedge \llbracket P \rrbracket\}$

(iii) $\llbracket \mathbb{P} C^{\mathbb{P} T} \rrbracket \quad=_{d f} \quad\{z: \mathbb{P} T \mid \forall x: T \bullet x \in z \Rightarrow x \in \llbracket C \rrbracket\}$

(iv) $\llbracket C_{1}{ }^{\mathbb{P} T_{1}} \times C_{2}{ }^{\mathbb{P} T_{2}} \rrbracket \quad={ }_{d f} \quad\left\{z: T_{1} \times T_{2} \mid z .1 \in \llbracket C_{1} \rrbracket \wedge z .2 \in \llbracket C_{2} \rrbracket\right\}$

(v) $\llbracket \mathbb{N} \rrbracket \quad={ }_{d f} \quad\{z: \mathbb{N} \mid z=z\}$

(vi) $\quad \llbracket\left[\cdots l_{i} \in C_{i}^{T_{i}} \cdots\right] \rrbracket \quad{ }_{d f} \quad\left\{z:\left[\cdots l_{i}: T_{i} \cdots\right] \mid\right.$ $\left.\cdots \wedge z . l_{i} \in \llbracket C_{i} \rrbracket \wedge \cdots\right\}$

(vii) $\llbracket \lambda x \in C^{\mathbb{P} T_{1}} \bullet t^{T_{2}} \rrbracket \quad=_{d f} \quad\left\{z: T_{1} \times T_{2} \mid\right.$

$$
z .1 \in \llbracket C \rrbracket \wedge z .2=\llbracket t[x / z .1] \rrbracket\}
$$

\begin{tabular}{|c|c|c|c|}
\hline (i) & $\llbracket x \rrbracket$ & $={ }_{d f}$ & $x$ \\
\hline (ii) & $\llbracket n \rrbracket$ & $={ }_{d f}$ & $n$ \\
\hline (iii) & $\llbracket t . l \rrbracket$ & $={ }_{d f}$ & $\llbracket t \rrbracket . l$ \\
\hline (iv) & $\llbracket\left\langle\cdots l_{i} \Rightarrow t_{i} \cdots\right\rangle \rrbracket$ & $={ }_{d f}$ & $\downarrow \cdots l_{i} \Rightarrow \llbracket t_{i} \rrbracket$ \\
\hline$(v)$ & $\llbracket t .1 \rrbracket$ & $={ }_{d f}$ & $\llbracket t \rrbracket .1$ \\
\hline (vi) & $\llbracket t .2 \rrbracket$ & $={ }_{d f}$ & $\llbracket t \rrbracket .2$ \\
\hline (vii) & $\llbracket\left(t_{0}, t_{1}\right) \rrbracket$ & $={ }_{d f}$ & $\left(\llbracket t_{0} \rrbracket, \llbracket t_{1} \rrbracket\right)$ \\
\hline (viii) & $\llbracket$ let $x==t_{0}$ in $t_{1} \rrbracket$ & $={ }_{d f}$ & $\llbracket t_{1} \rrbracket\left[x / \llbracket t_{0} \rrbracket\right]$ \\
\hline (ix) & $\llbracket\left(\lambda z \in C \bullet t_{1}\right) t_{0} \rrbracket$ & $={ }_{d f}$ & $\llbracket t_{1} \rrbracket\left[z / \llbracket t_{0} \rrbracket\right]$ \\
\hline$(x)$ & $\llbracket t \uparrow T \rrbracket$ & $={ }_{d f}$ & $\llbracket t \rrbracket \mid T$ \\
\hline
\end{tabular}

\subsection{Other terms}

In addition to the sets, which are translated above, there are two new forms of term in Z. In full the translation is:

Lemma 5.1. $\llbracket P[x / t] \rrbracket=\llbracket P \rrbracket[x / \llbracket t \rrbracket]$

Proof. Variables are unchanged by the translation and this is sufficient.

We will use this property without further reference in the sequel. 


\subsection{Correctness of the translation}

The syntax of the systems $\mathrm{Z}$ and $\mathrm{Z}_{C}$ are both given in two parts: a proto-syntax equipped with rules for type assignment and propositionhood. Our translation supposes that the $\mathrm{Z}$ expressions it considers are well-formed, but yields expressions which are prima facie only in the proto-syntax of $Z_{C}$. It is incumbent upon us to show that the translation preserves syntactic well-formedness. This is the content of the following proposition.

\section{Proposition 5.2.}

(i) If $\Gamma \triangleright_{Z} P$ prop then $\Gamma \triangleright_{C} \llbracket P \rrbracket$ prop

(ii) If $\Gamma \triangleright_{Z} t: T$ then $\Gamma \triangleright_{C} \llbracket t \rrbracket: T$

Proof. By simultaneous induction on the structure of the antecedent derivations. We give one case for illustration.

Case rule $\left(Z_{\exists}\right)$ : we have $\Gamma \triangleright_{C} \llbracket C \rrbracket: \mathbb{P} T$ ex hypothesi from which, by lemma 2.3(iii), we may conclude that $\Gamma, z: T \triangleright_{C} \llbracket C \rrbracket: \mathbb{P} T$ for fresh $z$. From this and the instance of axiom $\left(C_{x}\right) \Gamma, z: T \triangleright_{C} z: T$ we have $\Gamma, z: T \triangleright_{C} z \in \llbracket C \rrbracket$ prop by rule $\left(C_{\in}\right)$. From this and $\Gamma, z: T \triangleright_{C} \llbracket P \rrbracket$ prop, which follows ex hypothesi, we may conclude that $\Gamma, z: T \triangleright_{C} z \in \llbracket C \rrbracket \wedge \llbracket P \rrbracket$ prop by (derived) rule $\left(C_{\wedge}\right)$. Then by rule $\left(C_{\exists}\right)$ we have $\Gamma \triangleright_{C} \exists z: T \bullet z \in \llbracket C \rrbracket \wedge \llbracket P \rrbracket$ prop which is $\Gamma \triangleright_{C} \llbracket \exists z \in C \bullet P \rrbracket$ prop as required.

As a corollary we have the soundness of the type assignment system in ZF. We temporarily write $\llbracket-\rrbracket$ for the composition $\llbracket-\rrbracket_{C} \circ \llbracket-\rrbracket_{Z}$.

Corollary 5.3. If $\Gamma \triangleright_{Z} t: T$ then $\llbracket \Gamma \rrbracket \vdash_{Z F} \llbracket t \rrbracket \in \llbracket T \rrbracket$

Proof. Compose propositions 3.1 and 5.2.

Next we have the relative soundness result for the logic.

Proposition 5.4. If $\Gamma^{-} ; \Gamma^{+} \vdash_{Z} P$ then $\Gamma^{-} ; \llbracket \Gamma^{+} \rrbracket \vdash_{C} \llbracket P \rrbracket$

Proof. By induction on the structure of the antecedent derivation. We provide one case for illustration.

Case $\left(\exists^{+}\right)$:

Ex hypothesi we have $\llbracket P \rrbracket[z / \llbracket t \rrbracket]$ and $\llbracket t \rrbracket \in \llbracket C \rrbracket$. By (derived) rule $\left(\wedge^{+}\right)$we have $(z \in \llbracket C \rrbracket \wedge \llbracket P \rrbracket)[z / \llbracket t \rrbracket]$ and, using proposition $2.9, \llbracket t \rrbracket: T$ for some $T$. Hence, by rule $\left(\exists^{+}\right), \exists z: T \bullet z \in \llbracket C \rrbracket \wedge \llbracket P \rrbracket$ which is $\llbracket \exists z \in C^{\mathbb{P} T} \bullet P \rrbracket$ as required.

Finally, as a corollary, we have soundness for the logic in ZF.

Corollary 5.5. If $\Gamma \vdash_{Z} P$ then $\llbracket \Gamma \rrbracket \vdash_{Z F} \llbracket P \rrbracket$

Proof. Combine propositions 5.4 and 3.2.

This together with corollary 5.3 completes the process of modelling $\mathrm{Z}$ in $\mathrm{ZF}$.

\section{Conclusions}

We have introduced a specification $\operatorname{logic} Z_{C}$ which is sound with respect to a simple set-theoretic semantics. We have gone on to demonstrate that the basic apparatus of the specification language $\mathrm{Z}$ can be defined within $\mathrm{Z}_{C}$. As a result the extended logic is both conservative over $Z_{C}$, and, as a corollary of this, 
consistent. Much of the technical development concerns the interplay between the type systems and the logic proper: we have spelled out what it means for terms to be well-typed and, correspondingly, what it means for a proposition to be well-formed. Inference is shown to preserve these properties, and we have established that the translation of the more elaborate system into $Z_{C}$ preserves both well-formation and inference properties. Our logics contrast with the current draft of the $\mathrm{Z}$ standard (Annex $\mathrm{F}$ of [Nic95]) in several respects. That system should, but does not, preserve well-formation of terms and propositions, there exists no soundness proof with respect to the independently given semantics and there is no clear technical connection which links the logic of well-formation with the logic proper. In these regards, in particular, our formulation is an improvement.

We have, however, not gone far enough. The extended logic described here is still impoverished in several important respects, notably the absence of a calculus for schemas. The standard logic [Nic95] is also incomplete in this area. It is the purpose of the companion paper "Revising Z: Part II - logical development" [HeR99] to make good these omissions and to continue the critique we have begun here.

\section{Acknowledgements}

We would like to thank the Department of Computer Science at the University of Waikato, New Zealand, the Centre for Discrete Mathematics and Theoretical Computer Science, New Zealand, the Royal Society of Great Britain, and the EPSRC (grant number GR/L57913) for financial assistance which has supported the development of this research. We are most grateful to Doug Goldson, Lindsay Groves, Ian Toyn, Ray Turner and Mark Utting for many useful discussions. We are particularly grateful to the Editor-in-Chief, Cliff Jones, for his help and encouragement in the preparation of the final version of this paper. Our final thanks go to one of the referees, who performed the most thorough and fruitful task of refereeing that we have ever experienced.

\section{References}

[BrM96] Brien, S. and Martin, A.: A tutorial of proof in standard Z. Technical report, Technical monograph PRG-120, University of Oxford, 1996.

[Bri95] Brien, S.: A model and logic for generically typed set theory (Z). Technical report, (draft) D. Phil. thesis, University of Oxford, 1995.

[Hen98] Henson, M. C.: The standard logic for Z is inconsistent. Formal Aspects of Computing Journal, 10(3): 243-247, 1998.

[HaM97] Hall, J. and Martin, A.: $\mathscr{W}$ reconstructed. In Proceedings ZUM '97, LNCS 1212, pages 115-134. Springer, 1997.

[HeR99] Henson, M. C. and Reeves, S.: Revising Z: Part II - logical development. Formal Aspects of Computing Journal, 11(4): 381-401, 1999.

[KSW96] Kolyang, Santen, B. and Wolff, B.: A structure preserving encoding of $Z$ in Isabelle/HOL. In Proceedings Formal Methods Europe. LNCS Vol. 1051, Springer Verlag, 1996.

[Mar97] Martin, A.: Approaches to proof in Z. Technical report, Technical Report TR97-34, SVRC, University of Queensland, 1997.

[Mar98] Martin, A.: A revised deductive system for Z. Technical report, Technical Report TR9821, SVRC, University of Queensland, 1998.

[Nic95] Nicholls, J. (ed.): Z Notation: Version 1.2. Z Standards Panel, 1995. 
[Spi88] Spivey, J. M.: Understanding Z: A specification language and its formal semantics. C.U.P., 1988.

[Spi92] Spivey, J. M.: The $Z$ notation: A reference manual. Prentice Hall, 1992.

[Toy97] Toyn, I. (ed.): $Z$ Notation: Draft 0.8. Unpublished draft, 1997.

[WoB92] Woodcock, J. and Brien, S.: $\mathscr{W}:$ A logic for $Z$. In Proceedings of ZUM '91, 6th Conf. on Z. Springer Verlag, 1992.

[WoD96] Woodcock, J. and Davies, J.: Using Z: Specification, Refinement and Proof. Prentice Hall, 1996.

\section{Note added in proof}

Since this paper was accepted for publication, the draft Z standard [Nic95] has been superseded by the Final Committee Draft Standard [Toy99]. Our comments regarding semantics remain germane. On the other hand, the $\mathrm{Z}$ standards panel has decided to remove the logic entirely, rather than to correct it. As a result, our paper now becomes, at least in part, a work of advocacy in favour of a logic for $\mathrm{Z}$.

[Toy99] Toyn, I. (ed.): Z Notation: Final Committee Draft, CD 13568.2,

ftp://ftp.york.ac.uk./hise_reorts/cadiz/ZSTAN/fcd.ps.gz, 1999

Received March 1998

Accepted in revised form April 1999 by C. B. Jones 